\title{
Non-adiabaticity and other aspects of polarisation in positron-molecule scattering
}

\author{
Brian K Elza†, Thomas L Gibson $\ddagger$, Michael A Morrison $\dagger$ and Bidhan \\ C Sahat \\ $\uparrow$ Department of Physics and Astronomy, University of Oklahoma, Norman, Oklahoma \\ 73019, USA \\ ¥Department of Physics, Texas Technical University, Lubbock, Texas 79409, USA
}

Received 7 June 1988, in final form 26 August 1988

\begin{abstract}
We have investigated various aspects of polarisation/correlation effects at low energies for the prototypical positron- $\mathrm{N}_{2}$ system. First, we have found that, like positron- $\mathrm{H}_{2}$ cross sections, those for this system are highly sensitive to terms in the (adiabatic) polarisation potential that explicitly depend on the sign of the charge of the projectile. Insight into the nature of these sign-dependent distortions is afforded by three-dimensional graphs of polarised $\mathrm{N}_{2}$ density functions. Second, we have explored and characterised nonadiabatic (correlation) effects in this system using a simple two-parameter model. We have found that the onset of these effects in the near-target region in positron scattering is much more gradual than in electron scattering. By including in scattering calculations adiabatic and non-adiabatic effects, we obtain theoretical positron- $\mathrm{N}_{2}$ cross sections in good agreement with recent experimental data.
\end{abstract}

\section{Introduction}

The last few years have seen a dramatic increase in research on low-energy positronmolecule scattering (see, for example, the review by Armour (1988)). This activity is in part due to new technology for producing improved positron beams (Canter and Mills 1982). Nevertheless, there remain considerable uncertainties in measured values of positron-molecule cross sections at collision energies below a few eV (cf Hoffman et al 1982, Charlton et al 1983, Sueoka and Mori 1984), and fundamental questions plague the calculation of theoretical positron scattering cross sections at these energies.

Below the threshold for positronium formation, the most serious of these questions concerns polarisation and (short-range) correlation effects. Although these effects significantly influence low-energy cross sections, their inclusion in computational methods via accurate yet tractable approximations is problematical.

Two groups have recently initiated studies based on ab initio treatments of these effects. Armour and collaborators (Armour 1984, 1985, Armour and Baker 1985, 1986, 1987) have published a series of increasingly sophisticated variational calculations of positron- $\mathrm{H}_{2}$ collisions based on the Kohn method. Tennyson (1986) and Tennyson and Morgan (1987) have applied the $R$-matrix method to positron scattering from $\mathrm{H}_{2}$, $\mathrm{N}_{2}$ and $\mathrm{CO}$.

An alternative to these $a b$ initio theories is the use of a physically motivated model potential. At large distances from the target, the form of such a potential is simple and familiar: for a projectile at position $\left(r_{q}, \theta_{q}\right)$ (in a body-fixed coordinate system), 
the asymptotic polarisation potential is an analytic function of the spherical and non-spherical polarisabilities of the target, $\alpha_{0}$ and $\alpha_{2}$, i.e.,

$$
V_{\text {pol }}^{ \pm}\left(r_{q}, \theta_{q}\right) \underset{r_{q} \rightarrow \infty}{\longrightarrow}-\frac{\alpha_{0}}{2 r_{q}^{4}}-\frac{\alpha_{2}}{2 r_{q}^{4}} P_{2}\left(\cos \theta_{q}\right) \text {. }
$$

This form pertains to both positron (plus superscript) and electron (minus superscript) scattering; it is the asymptotic limit of the polarisation potential as calculated in second-order perturbation theory (Castillejo et al 1960; ch 2 of Gibson 1982). Hence this form is independent of the sign of the charge of the projectile.

But nearer the target, the simple form (1) does not accurately characterise polarisation effects, the second-order perturbation approximation is invalid, and $V_{\text {pol }}^{ \pm}\left(r_{q}, \theta_{q}\right)$ does depend on the sign; i.e., the polarisation potential for an incident positron differs from that for an incident electron. In 1984, Morrison et al demonstrated the importance of this dependence for positron- $\mathrm{H}_{2}$ cross sections and calculated a sign-dependent potential which is accurate beyond second order in the projectile-target interaction by treating the projectile's position adiabatically and determining via the linear variational method the decrease in the system energy due to polarisation of the molecule.

The importance of explicitly allowing for the sign of the projectile's charge in generating a polarisation potential for positron scattering calculations has been an issue because of the widespread use of (model) polarisation potentials that were determined not for the positron-molecule system, but for the corresponding electronmolecule system. This practice continues to the present day. Thus Jain (1986a, b) and Gianturco et al (1987) have suggested that the sensitivity to the sign of the projectile seen by Morrison et al in positron- $\mathrm{H}_{2}$ cross sections may be a fluke: that $\mathrm{H}_{2}$, nearly spherical and with only two bound electrons, is somewhat atypical, and that for heavier targets a treatment of polarisation that does not distinguish between electrons and positrons as projectiles may be approximately valid. Investigation of this question for a typical positron-molecule system, positron- $\mathrm{N}_{2}$, is one focus of the present study.

Our second area of concern-non-adiabaticity-becomes important within the molecular charge cloud. Here the adiabatic approximation breaks down completely and non-adiabatic effects become important: particularly correlation effects. Several investigators have developed and tested methods, ab initio and model-based, for including such effects in electron scattering (see Morrison et al 1987 and references therein, Schneider and Collins 1983, Huo et al 1987). Some positron-molecule studies use these potentials without change (see references in table II of Morrison 1986).

However, the importance in the near-target region of higher-order perturbation terms, which are senstive to the nature of the projectile, raises questions about the use of electron-molecule polarisation potentials in positron-molecule scattering calculations. Moreover, some methods for treating non-adiabaticity, such as the nonpenetrating approximation (Temkin 1957), which we have applied to the electron- $\mathrm{H}_{2}$ (Gibson and Morrison 1984) and electron- $\mathrm{N}_{2}$ (Morrison et al 1987) systems, seem inappropriate on physical grounds to positron-molecule scattering.

A central finding of the present study (see $\$ 4$ ) concerns the onset of these nonadiabatic effects as the projectile nears the target. To characterise this onset, we have used a two-parameter cut-off function that smoothly eliminates the adiabatic polarisation potential as $r_{q} \rightarrow 0$. By comparing our calculated cross sections with measured values, we can determine optimum parameters in this function; significantly, the values we obtain contrast strikingly with those required for electron- $\mathrm{N}_{2}$ scattering. (This strategem is similar in spirit to that used in recent positron-atom studies (Nakanishi 
and Schráder 1986a, b)). We offer in $\S 4$ an explanation based on the underlying physics of positron and electron collisions for the differences in the onset of nonadiabatic phenomena in the two systems.

\section{Theory}

For the scattering processes of interest here, the fixed-nuclei approximation is valid (Temkin and Vasavada 1967), so we work in a body-fixed (BF) reference frame (Lane 1980 ) and choose the origin of a single-centre coordinate system at the centre of mass of the molecule and the $z$ axis along the internuclear axis. We denote by $\boldsymbol{r}_{\mathrm{m}}, \boldsymbol{R}_{\alpha}$, and $\boldsymbol{r}_{q}$ the spatial coordinates of the molecular electrons, the target nuclei, and the scattering projectile. A semi-colon connotes parametric dependence on whatever variables appear after it.

Thus for a projectile at $\boldsymbol{r}_{q}$ scattering from a molecule with $N_{\mathrm{e}}$ target electrons at locations collectively denoted by $\boldsymbol{r}_{\mathrm{m}}$ and with $N_{\mathrm{n}}$ nuclei with charge $Z_{\alpha}$ fixed at $\boldsymbol{R}_{\alpha}$, the Coulomb potential is

$$
V_{\text {Coul }}^{ \pm}\left(\boldsymbol{r}_{\mathrm{m}} ; \boldsymbol{r}_{q}, \boldsymbol{R}_{\alpha}\right)=\mp \sum_{i=1}^{N_{e}} \frac{1}{\left|\boldsymbol{r}_{q}-\boldsymbol{r}_{i}\right|} \pm \sum_{\alpha=1}^{N_{\mathrm{n}}} \frac{Z_{\alpha}}{\left|\boldsymbol{r}_{q}-\boldsymbol{R}_{\alpha}\right|} .
$$

In the body-frame fixed-nuclei scattering equations, which result from projecting out of the time-independent Schrödinger equation for the positron-molecule system the (Born-Oppenheimer) ground electronic state of the target (see Morrison 1988), the effective positron-molecule interaction potential $V_{\text {int }}^{+}\left(\boldsymbol{r}_{q} ; R\right)$ is the sum of static and polarisation terms,

$$
V_{\mathrm{int}}^{+}\left(\boldsymbol{r}_{q} ; R\right)=V_{\mathrm{st}}^{+}\left(\boldsymbol{r}_{q} ; R\right)+V_{\mathrm{pol}}^{+}\left(\boldsymbol{r}_{q} ; R\right) .
$$

The electrostatic term is the average (the integral with respect to electronic coordinates $\left.r_{\mathrm{m}}\right)$ of the Coulomb interaction (2) over $\psi_{0}\left(\boldsymbol{r}_{\mathrm{m}} ; R\right)$, the ground electronic state of the target at internuclear separation $R$, i.e.,

$$
V_{\mathrm{st}}^{+}\left(\boldsymbol{r}_{q} ; R\right)=\left\langle\psi_{0}\left|V_{\text {Coul }}^{+}\right| \psi_{0}\right\rangle
$$

To characterise the polarisation potential $V_{\mathrm{pol}}^{+}\left(\boldsymbol{r}_{q} ; R\right)$ it is useful to consider three 'regions' of configuration space. These regions can be loosely delineated by $r_{q}$, the distance of the positron from the molecular centre of mass. In the asymptotic region the polarisation potential reduces to the simple sign-independent analytic form (1). In the intermediate region, at values of $r_{q}$ outside the molecular charge cloud but near enough to it that equation (1) is invalid, one can obtain an accurate polarisation potential for low-energy collisions (impact energies less than about $10 \mathrm{eV}$ ), by assuming (Morrison and Hay 1979, Truhlar et al 1979) that the target responds adiabatically to the projectile-i.e., that the system is accurately represented by an adiabatic Hamiltonian $\hat{\mathscr{H}}_{\mathrm{AD}}^{ \pm}$, which is the sum of the (Born-Oppenheimer) electronic Hamiltonian of the target and the Coulomb interaction (2) with the fixed radial coordinate $r_{q}$ treated as a parameter.

The present positron- $\mathrm{N}_{2}$ study uses such a treatment. At each internuclear separation $R$, the seven $\mathrm{N}_{2}$ molecular orbitals $\left(1 \sigma_{\mathrm{g}}, 1 \sigma_{\mathrm{u}}, 2 \sigma_{\mathrm{g}}, 2 \sigma_{\mathrm{u}}, 3 \sigma_{\mathrm{g}}, 1 \pi_{\mathrm{u}}\right)$ are allowed to fully relax in the presence of a point charge whose location $\boldsymbol{r}_{q}$ is 'frozen'. From the 
resulting distorted molecular orbitals we construct the polarised electronic wavefunction $\psi_{0}^{(p)}\left(\boldsymbol{r}_{\mathrm{m}} ; \boldsymbol{r}_{q}, R\right)$ of the target. The adiabatic polarisation potential at each $R$ is just the difference between the expectation values of $\hat{\mathscr{H}}_{\mathrm{AD}}^{ \pm}$with respect to the polarised and unpolarised target wavefunctions, i.e.,

$$
V_{\mathrm{AD}}^{ \pm}\left(\boldsymbol{r}_{q} ; R\right)=\left\langle\psi_{0}^{(p)}\left|\hat{\mathscr{H}}_{\mathrm{AD}}^{ \pm}\right| \psi_{0}^{(p)}\right\rangle-\left\langle\psi_{0}\left|\hat{\mathscr{H}}_{\mathrm{AD}}^{ \pm}\right| \psi_{0}\right\rangle
$$

In the region nearest the target, within the molecular charge cloud, the adiabatic polarisation potential improperly correlates the projectile and the bound electrons: the adiabatic approximation overestimates correlation effects, leading to a polarisation potential that is too attractive. In this region, short-range correlation (e.g. virtual positronium formation) is important, and we must allow for non-adiabatic effects. (An adiabatic treatment in this region would be more appropriate were we determining a proton-molecule interaction potential, where the dominant short-range correlation effect is virtual hydrogen-atom formation.)

Our treatment of non-adiabaticity distinguishes in two ways the present positron polarisation potentials from our non-adiabatic electron potentials (Gibson and Morrison 1984, Morrison et al 1987). First, we do not use the non-penetrating approximation of Temkin (1957) to incorporate non-adiabatic effects in positron scattering potentials; this approximation, which essentially entails 'switching off' the Coulomb interaction between the projectile and the bound electrons whenever the former is inside the charge cloud of the latter, is inappropriate for positron collisions (see Morrison et al 1984). Second, we retain all multipoles in the polarisation potential. (For electron scattering, only the dipole term need be retained.)

Our primary interest in this study is the physical nature of non-adiabatic corrections to the adiabatic positron-molecule polarisation potential. We approximate these corrections by simply multiplying the polarisation potential, in either its asymptotic or adiabatic form (equations (1) or (5)), by a spherical cut-off function that depends on adjustable parameters $r_{\mathrm{c}}$ and $p$ :

$$
C\left(r_{q}\right)=1-\exp \left[-\left(r_{q} / r_{\mathrm{c}}\right)^{p}\right]
$$

Several investigators have used such functions in prior positron-molecule studies (see $\$ 2.1$ of Morrison et al (1984) and references in Morrison (1986)). This function accounts for non-adiabatic effects by smoothly eliminating the polarisation potential as $r_{q}$ decreases from $r_{\mathrm{c}}$ to zero. The 'power parameter' $p$ controls how sharply this potential vanishes, or alternatively, the size of the region of non-adiabaticity and the abruptness of the onset of non-adiabatic effects. Thus, within this scheme, $p$ characterises the coarse features of these effects.

Cut-off functions like equation (6) have been used to approximate non-adiabatic corrections in many electron-molecule scattering calculations (see Morrison 1983 and references therein). In such studies, the power parameter is usually chosen as $p=6$ and the 'cut-off radius' $r_{c}$ is adjusted to force calculated cross sections to replicate some experimentally determined feature of the system such as a shape resonance.

The choice $p=6$ in electron-molecule scattering reflects the suddenness of the onset of non-adiabatic effects as $r_{q}$ decreases to zero. The principal non-adiabatic correction is due to a velocity dependence in the polarisation potential; this dependence arises when a slow scattering electron 'falls into' the strongly attractive, short-range staticexchange potential well. The electron's local kinetic energy rapidly becomes comparable to that of the bound electrons, which completely invalidates the adiabatic 
hypothesis. The resulting correlation potential is considerably weaker near the origin than the adiabatic potential.

However, for positron-molecule scattering no comparable mechanism exists to cause the sudden onset of non-adiabatic effects. Because the positron-molecule static potential is repulsive, velocity-dependent non-adiabatic effects are much less important than in the corresponding electron-molecule system. Hence the dominant nonadiabatic effect is due to virtual positronium formation, which occurs gradually as the positron penetrates the molecular charge cloud.

Thus the physics of non-adiabaticity in positron-molecule scattering argues for using a small value of the power parameter-a value that permits a gradual onset of non-adiabatic effects. In our positron- $\mathrm{N}_{2}$ study $(\$ 4)$, we characterise the dominant non-adiabatic effects by varying this parameter, comparing calculated cross sections with experimental data.

\section{Computational considerations}

\subsection{Representation of the $\mathrm{N}_{2}$ target}

The ground-state $\left(\mathrm{X}^{1} \Sigma_{\mathrm{g}}^{+}\right) \mathrm{N}_{2}$ wavefunction appears in both the static and polarisation components of the positron- $\mathrm{N}_{2}$ interaction potential (equation (3)). We represented this function in the Hartree-Fock approximation, calculating molecular orbitals using a (10s6p2d/6s4p2d) basis of contracted nucleus-centred Cartesian Gaussian functions (Morrison and Hay 1979). This basis is an augmentation of a (9s5p1d/5s3p1d) basis, the additional (uncontracted) $\mathrm{s}, \mathrm{p}$ and $\mathrm{d}$ functions providing sufficient flexibility to accommodate distortions of the $\mathrm{N}_{2}$ charge density.

The static positron-molecule potential in the region of the target is strongly repulsive. Consequently the nature of the static and polarisation potentials outside this region, including their long-range functional forms, significantly influences the cross sections we want to determine. In the asymptotic region $\left(r_{q} \rightarrow \infty\right)$, the static potential (4) reduces to a simple analytic form (at internuclear separation $R$ ) whose Legendre projections

$$
v_{\lambda}^{\mathrm{st}}\left(r_{q} ; R\right)=\frac{2 \lambda+1}{2} \int_{0}^{\pi} V_{\mathrm{st}}^{+}\left(\boldsymbol{r}_{q} ; R\right) P_{\lambda}\left(\cos \theta_{q}\right) \sin \theta_{q} \mathrm{~d} \theta_{q}
$$

are proportional to the permanent multipole moments $c_{\lambda}(R)$, as

$$
v_{\lambda}^{\mathrm{st}}\left(r_{q} ; R\right) \underset{r_{q} \rightarrow \infty}{\longrightarrow} \pm \frac{c_{\lambda}(R)}{r_{q}^{\lambda+1}}
$$

where the plus sign corresponds to positron and the minus to electron scattering.

The values of the lowest three permanent moments, calculated from the static potential (based on our $(10 \mathrm{~s} 6 \mathrm{p} 2 \mathrm{~d} / 6 \mathrm{~s} 4 \mathrm{p} 2 \mathrm{~d})$ electronic function) at the equilibrium internuclear separation $R_{\mathrm{e}}=2.068 a_{0}$ are compared to experimental values in table 1 . We extracted the theoretical moments at $r_{q}=10.0 a_{0}$, by which point each Legendre projection $v_{\lambda}^{\text {st }}\left(r_{q} ; R\right)$ is given (to the stated precision) by its multipolar form $(8)$. Similarly, we determine the induced polarisabilities, $\alpha_{0}$ and $\alpha_{2}$ in the asymptotic form (1), from the calculated adiabatic polarisation potential $V_{\mathrm{AD}}^{ \pm}\left(r_{q} ; R\right)$ at $r_{q}=25.0 a_{0}$. the values of these moments also appear in table 1 . 
Table 1. Permanent and induced moments for $\mathrm{N}_{2}$ at $R=2.068 a_{0}$.

\begin{tabular}{|c|c|}
\hline \multicolumn{2}{|c|}{ Permanent moments } \\
\hline Theory $^{\mathrm{a}}$ & Experiment ${ }^{\mathrm{b}}$ \\
\hline $\begin{array}{l}c_{2}\left(R_{\mathrm{e}}\right)=0.91 e a_{0}^{2} \\
c_{4}\left(R_{\mathrm{e}}\right)=7.49 e a_{0}^{4} \\
c_{6}\left(R_{\mathrm{e}}\right)=20.68 e a_{0}^{6}\end{array}$ & $|1.04| \pm 0.07 e a_{0}^{2}$ \\
\hline \multicolumn{2}{|c|}{ Induced moments } \\
\hline Theory ${ }^{\mathrm{c}}$ & Experiment $^{d}$ \\
\hline $\begin{array}{l}\alpha_{0}(R)=11.42 a_{0}^{3} \\
\alpha_{2}(R)=3.37 a_{0}^{3}\end{array}$ & $\begin{aligned} 11.74 & \pm 0.004 a_{0}^{3} \\
2.96 & \pm 0.03 a_{0}^{3}\end{aligned}$ \\
\hline $\begin{array}{l}{ }^{\mathrm{a}} \text { Morrison et al (1 } \\
{ }^{\mathrm{b}} \text { Herzberg (1950), } \\
{ }^{\mathrm{c}} \text { Present study. M } \\
{ }^{\mathrm{d}} \text { Miller and Beder }\end{array}$ & $\begin{array}{l}\text { tracted at } r_{q}=1 \\
\text { al (1975). } \\
\text { extracted at } r_{q}= \\
\text { 8) and referenc }\end{array}$ \\
\hline
\end{tabular}

\subsection{Parameters of the scattering calculations}

In the fixed-nuclei approximation, the total cross section is the sum of contributions from independent calculations in various electron-molecule symmetries (see Morrison 1988 and references therein). These symmetries correspond to the irreducible representations of the point group of the molecule, and for $\mathrm{N}_{2}$ (the point group of which is $D_{\infty h}$ ) are defined by the projection $\Lambda$ of the projectile's orbital angular momentum on the internuclear axis and by the behaviour of the scattering function under inversion through the origin (gerade or ungerade). Positron-molecule cross sections decrease rapidly with increasing $\Lambda$, and for positron- $\mathrm{N}_{2}$ we can converge the total cross section (at energies less than or equal to $10 \mathrm{eV}$ ) to $1 \%$ by including contributions from $\Sigma_{g}, \Sigma_{u}, \Pi_{u}, \Pi_{g}, \Delta_{g}$ and $\Delta_{u}$ symmetries.

Within each symmetry, the most important convergence parameters are the number of partial waves (spherical harmonics $Y_{l}^{\Lambda}$ ) included in the single-centre expansion of the scattering function and the value $r_{\max }$ of the radial projectile coordinate at which we extract the scattering matrix. To satisfy our $1 \%$ convergence critieria, we require five partial waves in the $\Sigma$ symmetries $(\Lambda=0)$ and four in the $\Pi(\Lambda=1)$ and $\Delta(\Lambda=2)$ symmetries. In the Legendre expansion of the static potential (see equation (7)) we include $\left(\right.$ even- $\lambda$ ) projections up to $\lambda_{\max }=14$. We must propagate the scattering function to $r_{\max }=86.0 a_{0}$. We checked these values using the time-honoured method of including more partial waves (up to $l_{\max }=12$ ) and propagating further (to $r_{\max }=100.0 a_{0}$ ). All is well.

\section{Results}

To exhibit the nature of induced distortions of a molecular charge cloud by an incident projectile, electron or positron, we compare (in $\$ 4.1 .1$ ) neutral and polarised oneelectron $\mathrm{N}_{2}$ probability densities for various (fixed) projectile locations. These threedimensional graphs evince the sensititivity of polarisation effects to the sign of the 
charge of the projectile. Further insight into this response follows from examining individual molecular orbitals, important examples of which appear in \$ 4.1.2. The differences seen in these graphs of probability densities translate into differences in the polarisation potentials themselves, which are discussed in $\S 4.2$. These disparities, in turn, influence cross sections for positron- $\mathrm{N}_{2}$ scattering as calculated with various polarisation potentials; these scattering data are compared in $\$ 4.3$, where we reconsider non-adiabaticity, a central concern of this research.

\subsection{Variously polarised probability densities}

4.1.1. Total electron density functions. We calculate total one-electron probability densities of the (neutral or polarised) molecule from the Born-Oppenheimer electronic wavefunctions, $\psi_{0}\left(\boldsymbol{r}_{\mathrm{m}} ; R\right)$ or $\psi_{0}^{(p)}\left(\boldsymbol{r}_{\mathrm{m}} ; \boldsymbol{r}_{q}, \boldsymbol{R}\right)$ (see, for example, equation (5)). For a wavefunction polarised by a projectile at $\boldsymbol{r}_{q}$, this density function is an integral over all but one of the spatial variables of the bound electrons,

$$
\rho\left(\boldsymbol{r}_{1}\right)=\int \psi_{0}^{(p)^{*}}\left(\boldsymbol{r}_{\mathrm{m}} ; \boldsymbol{r}_{q}, \boldsymbol{R}\right) \psi_{0}^{(p)}\left(\boldsymbol{r}_{\mathrm{m}} ; \boldsymbol{r}_{q}, \boldsymbol{R}\right) \mathrm{d} \boldsymbol{r}_{N_{\mathrm{e}}} \mathrm{d} \boldsymbol{r}_{N_{\mathrm{e}}-1} \ldots \mathrm{d} \boldsymbol{r}_{2}
$$

To display the polarisation distortions, we show in this section graphs of the oneelectron density function evaluated in the $x z$ plane, i.e. we plot $\rho\left(r_{1}, \theta_{1}, \varphi_{1}=0\right)$ for $r_{1}$ from 0 to $5 a_{0}$ and for $\theta_{1}$ from 0 to $\pi$. Figure 1, for example, shows this function for the $X^{1} \Sigma_{\mathrm{g}}^{+}$ground state of the neutral (undistorted) $\mathrm{N}_{2}$ molecule. (To enable a clear visualisation of these density functions, their peaks (near the nitrogen nuclei at $\pm 1.034 a_{0}$ on the $z$ axis) have been clipped as necessary; hence the 'flat tops' in figure 1 and elsewhere.)

Figure 2 shows distortions of the total ground-state density due to a positron $(a)$ and an electron $(b)$; these projectiles were fixed at $z_{q}=1.5 a_{0}$, just beyond the nuclei. The distortions seen in these figures are not simple mirror images of one another; they differ in both form and extent. Thus, the extrusion of the positron-distorted density

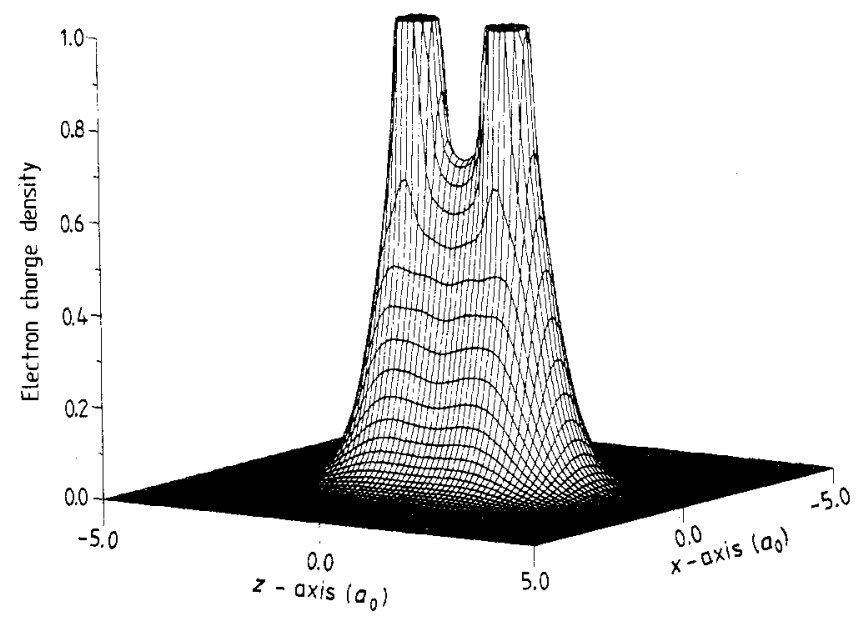

Figure 1. Total $\mathrm{N}_{2}$ electron density function (at $R=2.068 a_{0}$ ) in the $x z$ plane. In order to more clearly show changes in this function due to a fixed projectile (see figure 2 ), its peaks have been 'clipped' to a value of $\rho=1$. 

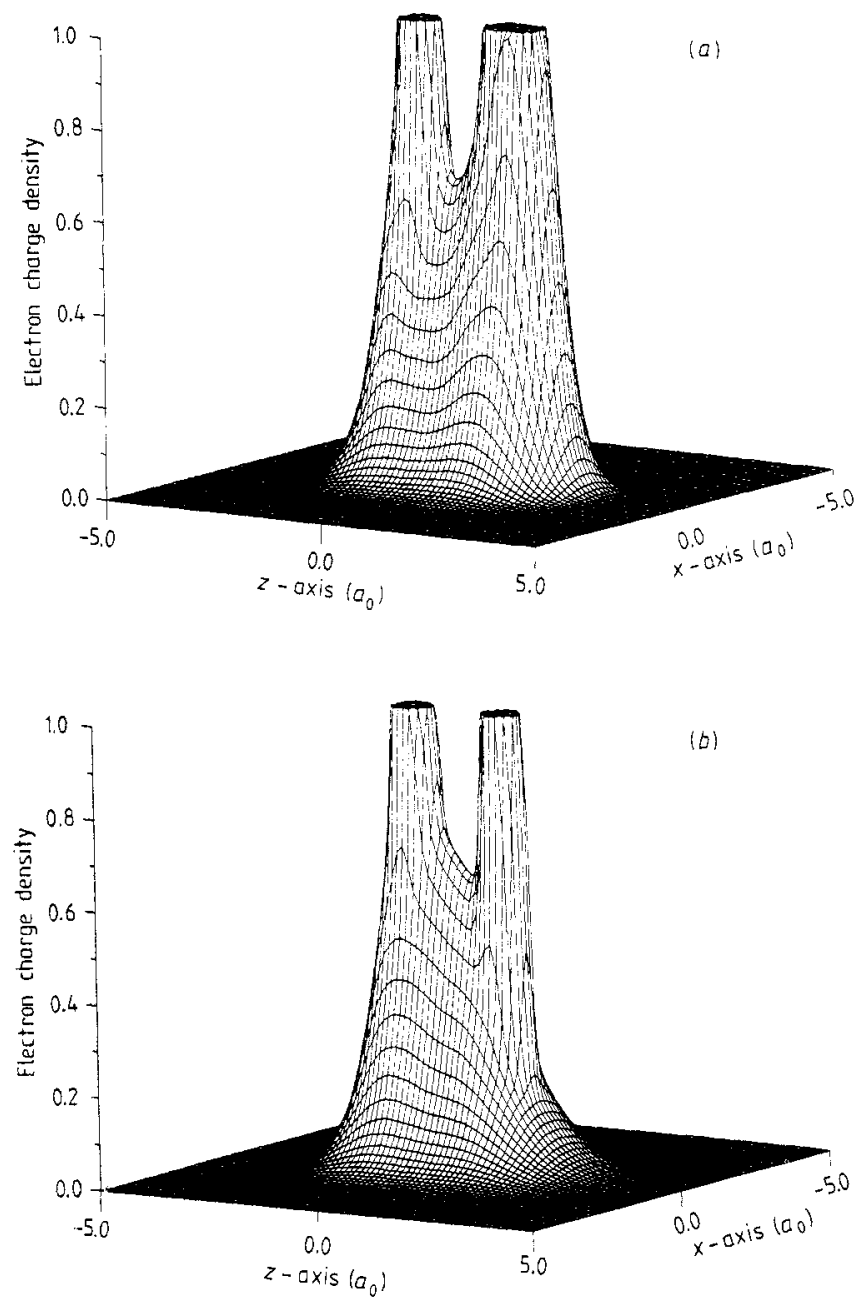

Figure 2. Total $\mathrm{N}_{2}$ density function polarised by ( $a$ ) a positron and $(b)$ an electron fixed at $z_{q}=1.5 a_{0}$.

in figure 2( $a$ ) towards the projectile, which is shaped by that projectile's (radial) field, is quite different from its counterpart in the electron-distorted density in figure $2(b)$.

4.1.2. Molecular orbital density functions. Because these total densities include all seven molecular orbitals of $\mathrm{N}_{2}$, they obscure some features of the distortions they are designed to illustrate. These features appear quite vividly, however, in graphs of the most diffuse molecular orbitals.

The $3 \sigma_{\mathrm{g}}$ and $2 \sigma_{\mathrm{u}}$ orbitals clearly exhibit the nature of these (adiabatic) polarisation distortions. The undistorted (neutral) densities for these orbitals appear in figures $3(a)$ and $4(a)$, respectively. Figures $3(b)$ and $(c)$ show the strikingly different distortions induced in the $3 \sigma_{\mathrm{g}}$ density by an electron and a positron at $z_{q}=1.5 a_{0}$. The lack of mirror symmetry in the polarised orbitals illustrated in this comparison also appears in the distorted $2 \sigma_{\mathrm{u}}$ orbitals in figures $4(b)$ and $(c)$.

As the distance of the (fixed) projectile from the molecule increases, the distortions of these densities decrease; ultimately, they regain their neutral shapes. In $\$ 4.2$ we 

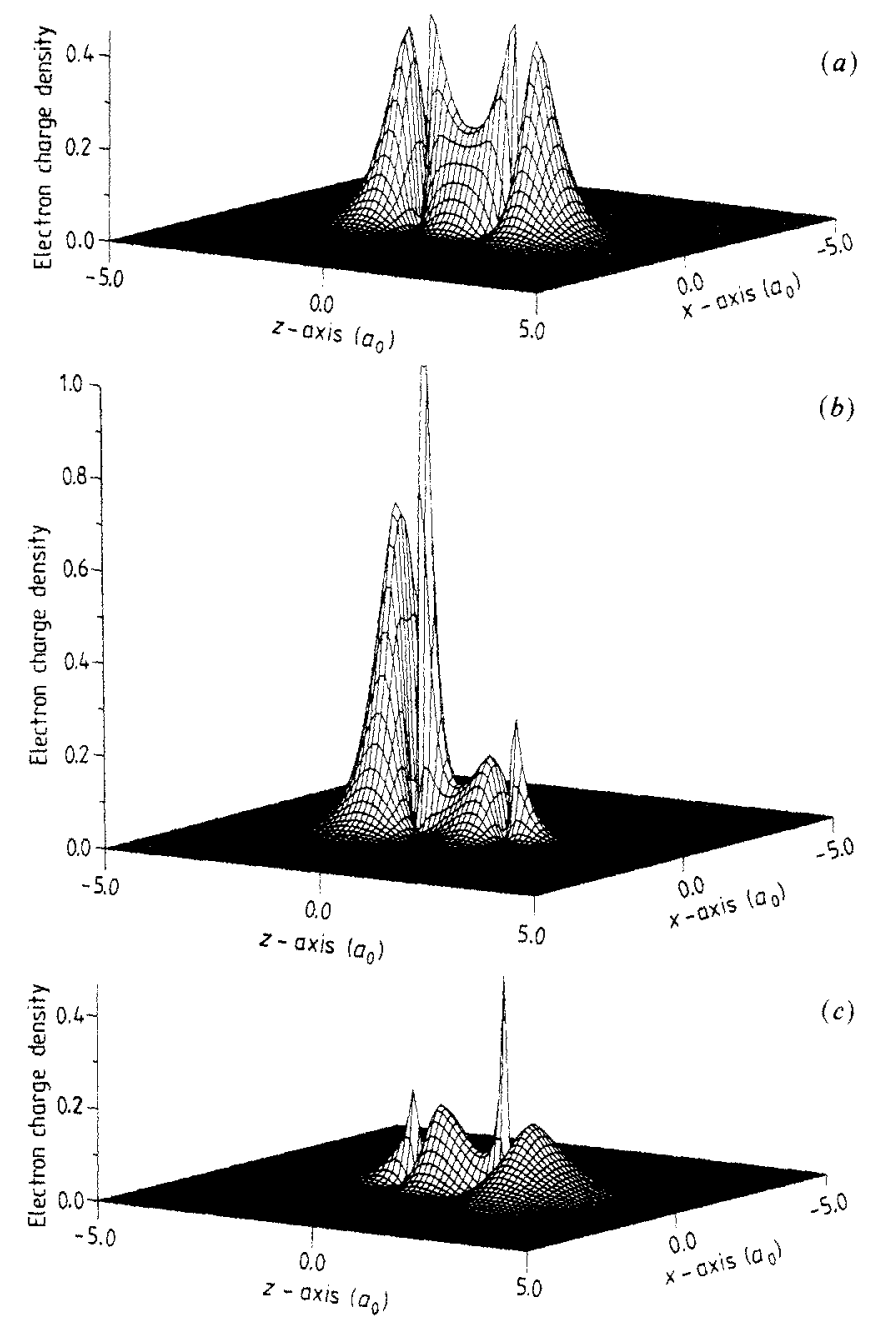

Figure 3. (a) The one-particle density function evaluated in the $x z$ plane for the $3 \sigma_{\mathrm{g}}$ molecular orbital of neutral $\mathrm{N}_{2}$. Also shown are the density functions that result when this orbital is polarised by $(b)$ a positron and $(c)$ an electron at $z_{q}=1.5 a_{0}$.

will discuss the effect on the polarisation potential of varying the projectile's location. The effect of this variation on the energies of the $3 \sigma_{\mathrm{g}}$ and $2 \sigma_{\mathrm{u}}$ orbitals is shown in figure 5. As expected, as the incident (negatively charged) electron enters the fringes of the molecular charge cloud, it enhances screening of the outermost electrons from the nucleus, causing their binding energies to decrease; precisely the opposite behaviour results from the incursion of an incident (positively charged) positron.

In any case, the way in which the positron- and electron-distorted orbitals correlate to the appropriate neutral energies (as $r_{q} \rightarrow \infty$ ) is strongly dependent on the sign of the charge. These comparisons reflect again the inadequacy of a second-order perturbative treatment of polarisation, an inherently sign-independent treatment, in the nonasymptotic region.

\subsection{Adiabatic and non-adiabatic polarisation potentials}

The energy lowerings due to the electron-and positron-distorted density functions in figure 2 -and their counterparts for other values of $r_{q}$-are the adiabatic polarisation 

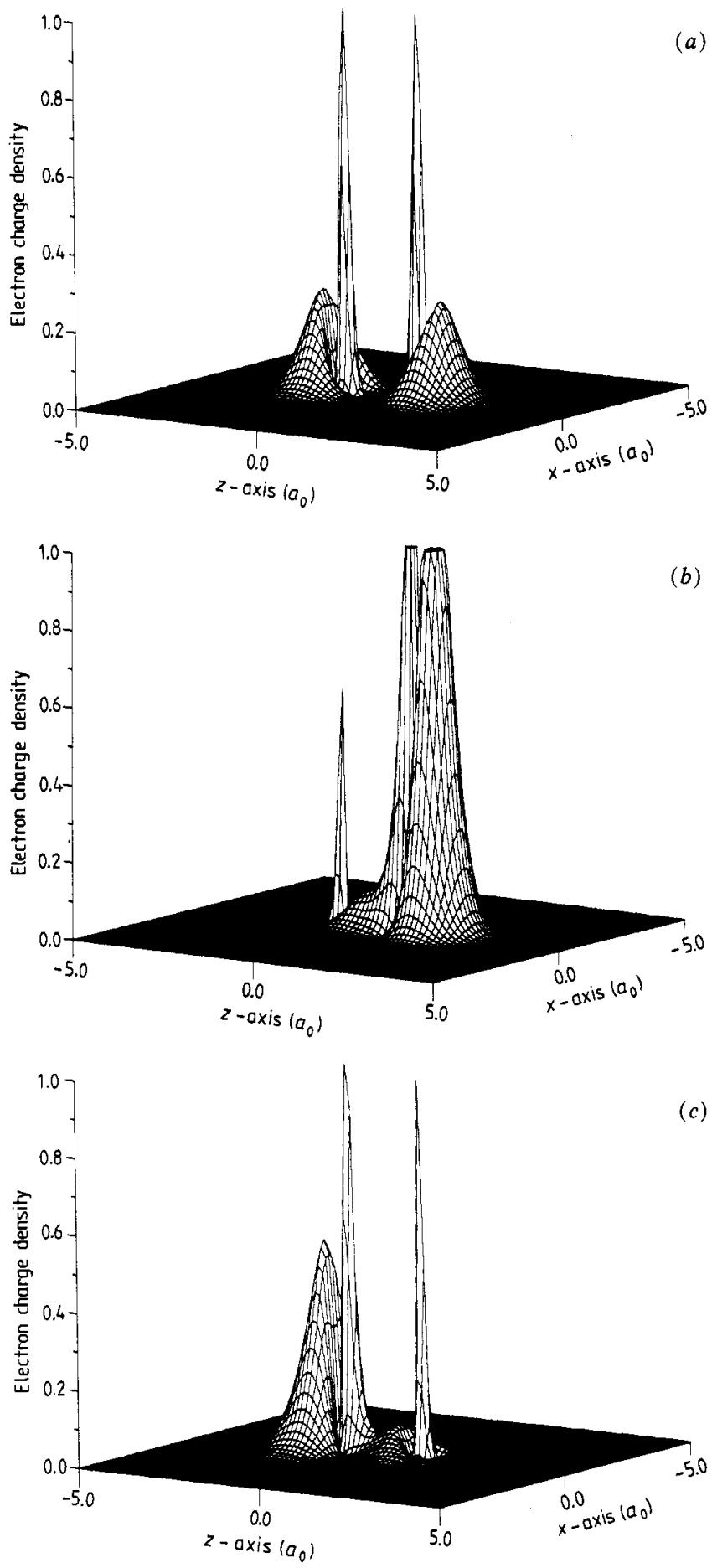

Figure 4. (a) The one-particle density function evaluated in the $x z$ plane for the $2 \sigma_{\mathrm{u}}$ molecular orbital of neutral $\mathrm{N}_{2}$. Also shown are the density functions that result when this orbital is polarised by $(b)$ a positron and $(c)$ an electron at $z_{q}=1.5 a_{0}$. 

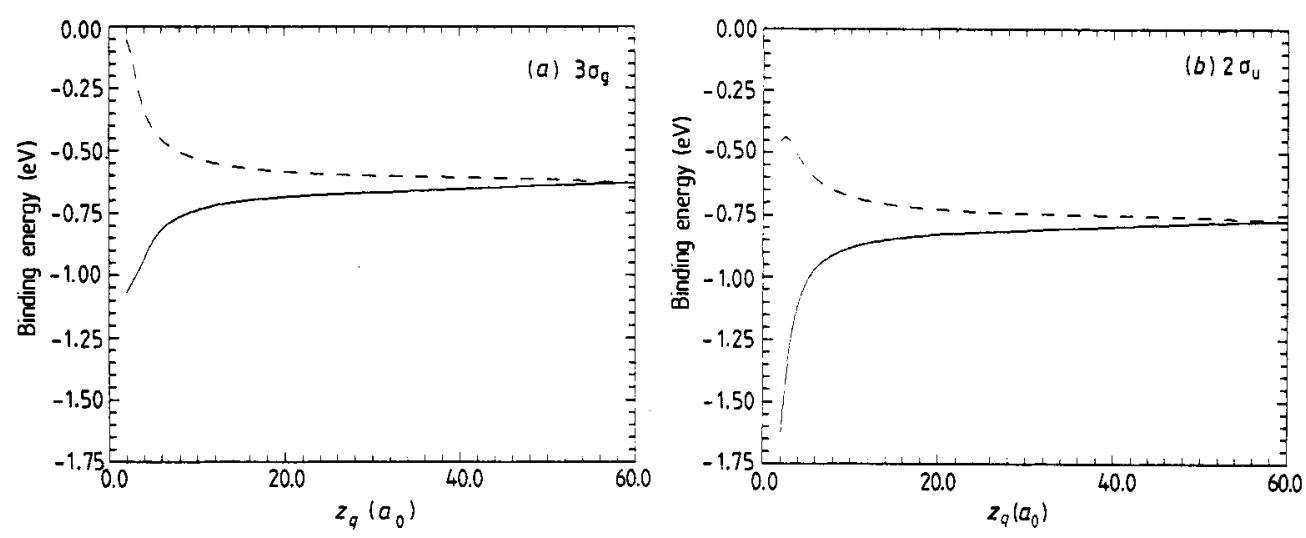

Figure 5. Energies of $\mathrm{N}_{2}$ molecular orbitals polarised by an incident electron (- - -) and by an incident positron (-) as a function of the distance of the (fixed) projectile along the $z$ axis. Shown are these 'correlation diagrams' for polarised orbitals that correlate (as $\left.r_{q} \rightarrow \infty\right)$ to the $(a) 3 \sigma_{\mathrm{g}}$ and $(b) 2 \sigma_{\mathrm{u}}$ orbitals of the neutral.

potentials (see equation (6)). We have designated these potentials by the acronyms ADELEC (for an incident electron) and ADPOS (for a positron). The two lowest-order Legendre projections (cf equation (7)) of these potentials are compared in figures $6(a)$ $\left(\lambda=0\right.$, the spherical projection) and $6(b)(\lambda=2)$. Note that as $r_{q} \rightarrow \infty$, the ADELEC and ADPOS potentials become identical: both reduce to the asymptotic form (1).

The similarities between the projections of these purely adiabatic potentials are deceptive; quite different potentials result when non-adiabatic effects are taken into account. To illustrate this point, we also show in figure 6 the projections of two non-adiabatic polarisation potentials: the 'better than adiabatic dipole' (BTAD) potential determined by Morrison et al (1987) for electron- $\mathrm{N}_{2}$ scattering and a cut-off ADPOS potential. The latter produces total positron- $\mathrm{N}_{2}$ cross sections that agree closely with measured values (see figure $10(b)$ ); we shall discuss this ADPOS potential in the next section.

These non-adiabatic potentials highlight an important difference between positronand electron-induced distortions of $\mathrm{N}_{2}$. This can most clearly be seen in the differences between the purely adiabatic and non-adiabatic potentials: within the approximate representation of non-adiabaticity inherent in both the BTAD and cut-off ADPOS models, these differences are 'the non-adiabatic polarisation potentials' due to, respectively, an electron and a positron. The spherical and $\lambda=2$ projections of these differences in figure 7 show that non-adiabatic effects are stronger and more extensive for a distorting electron than for a positron.

\subsection{Positron- $\mathrm{N}_{2}$ cross sections}

4.3.1. Total integrated cross sections. Our procedure for approximating the effects of non-adiabaticity-multiplying the adiabatic (or asymptotic) polarisation potential by the spherical cut-off function (6)-facilitates study of the importance of these effects to cross sections, by simply varying the adjustable parameters in $C\left(r_{q}\right)$ : the power parameter $p$ and the cut-off radius $r_{\mathrm{c}}$. Figure 8, for example, shows the sensitivity of the total positron- $\mathrm{N}_{2}$ cross section as calculated with the ADPOS (figure $8(a)$ ) or ADELEC (figure $8(b)$ ) polarisation potentials to variations in the power parameter $p$. Figure 9 shows their sensitivity to variation of the cut-off radius $r_{\mathrm{c}}$. 

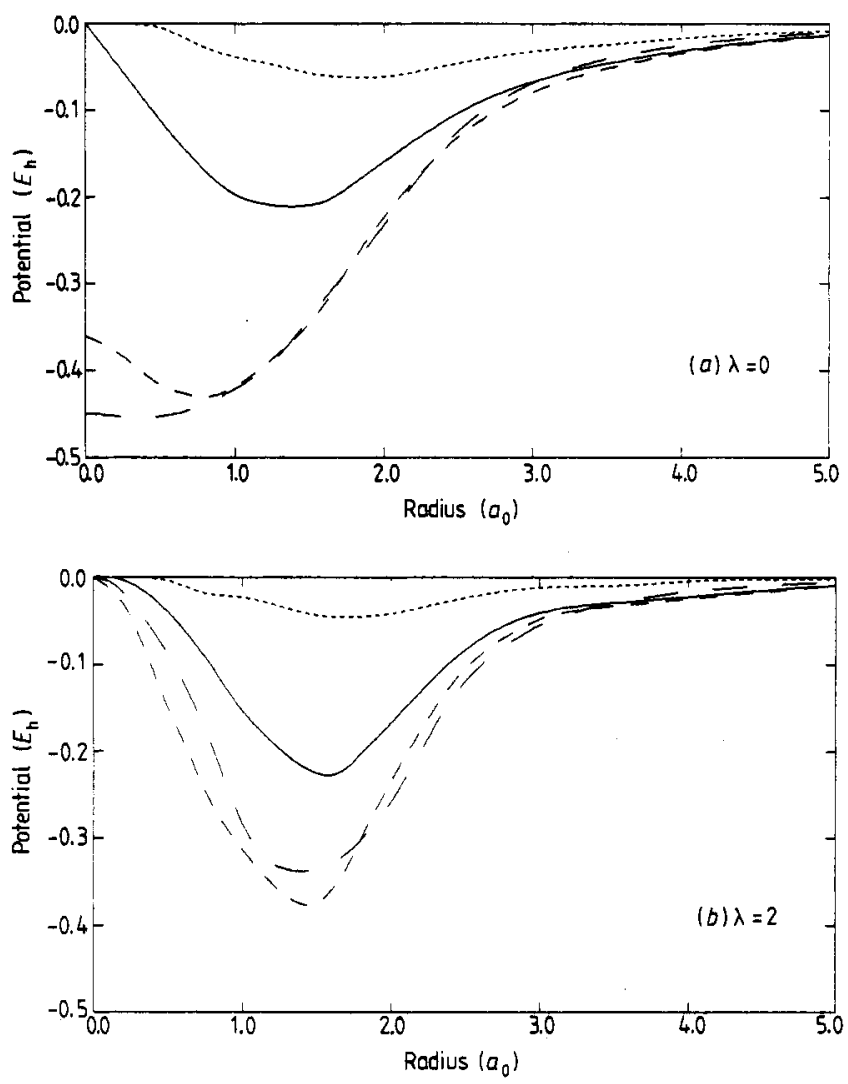

Figure 6. Adiabatic and non-adiabatic polarisation potentials for an incident electron and for an incident positron: ( $a$ ) the spherical Legendre projection, and $(b)$ the $\lambda=2$ projection. For each type of projectile, two curves are shown: purely adiabatic (long broken curve for an incident electron, short broken curve for a positron) and non-adiabatic (dotted curve for an electron, full curve for a positron). The latter potentials were chosen as those that most closely reproduce experimental cross section data for scattering from $\mathrm{N}_{2}$ : the nonadiabatic electron polarisation potential is the BTAD model of Morrison et al (1987); the non-adiabatic positron potential is the cut-off ADPOS model (with $r_{\mathrm{c}}=1.59 a_{0}$ and $p=1$ ) discussed in $\$ 4$.

Two points deserve emphasis. First, cross sections calculated using a positroninduced polarisation potential differ qualitatively from those determined with an electron-induced potential. For example, the cross sections in figure $8(b)$, calculated using the cut-off ADELEC potential, exhibit structure (Ramsauer minimum) not seen in the identically cut-off ADPOS results of figure $8(a)$ (nor in experimental data). Figure $9(a)$ shows, however, that we can force such a minimum into the ADPOS cross section by choosing an unrealistically large cut-off radius, $r_{\mathrm{c}}>2 a_{0}$.

This first observation confirms that the findings of Morrison et al (1984) regarding positron- $\mathrm{H}_{2}$ scattering also pertain to this typical heavier system: i.e., that the differences in variously polarised electron densities $(\$ 4.1)$ and in the polarisation potentials calculated from them $(\$ 4.2)$ translate into significant differences in scattering quantities.

Second, the total cross section is especially sensitive to the sharpness of onset of non-adiabatic effects as controlled by the power parameter $p$. In order to determine 

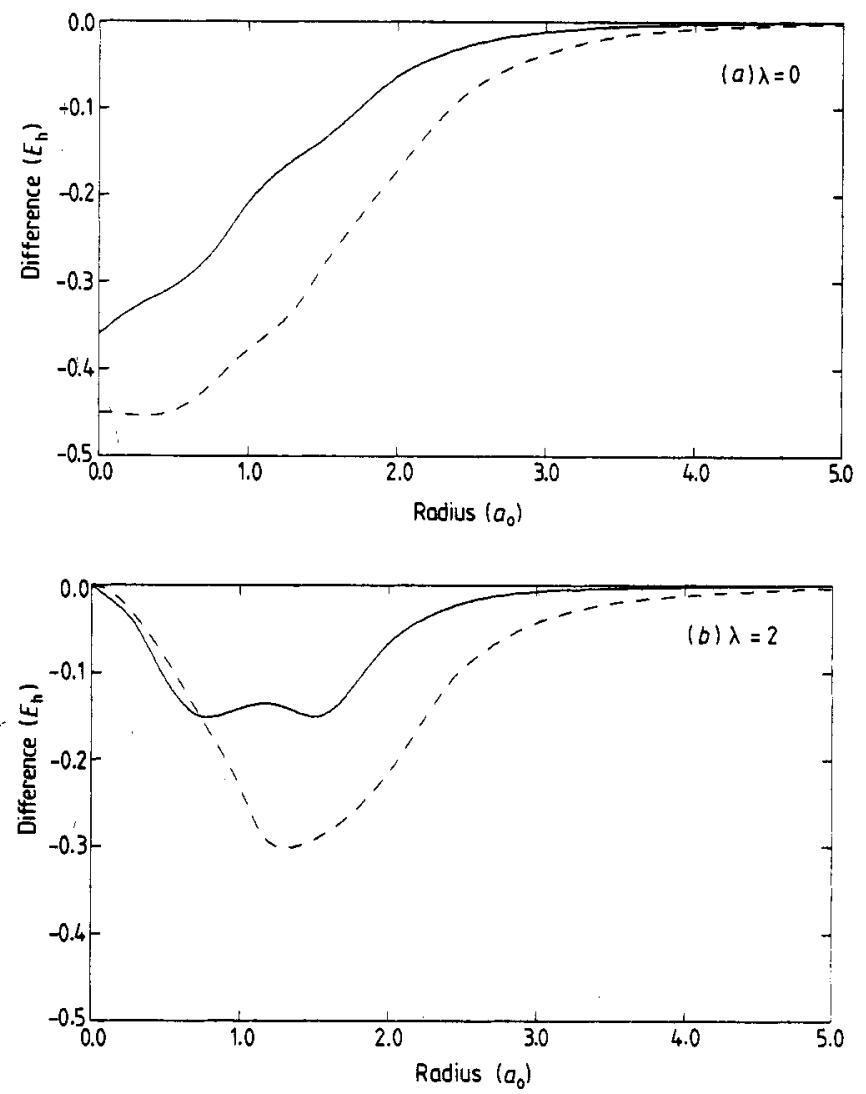

Figure 7. Model non-adabatic polarisation potentials for an incident electron (broken curves) and an incident positron (full curves): $(a)$ the spherical $(\lambda=0)$ and $(b) \lambda=2$ Legendre projections. In each case, these are the differences between the purely adiabatic potentials in figure 6 and their counterparts including non-adiabatic effects: the BTAD for an incident electron, the cut-off ADPOS for an incident positron.
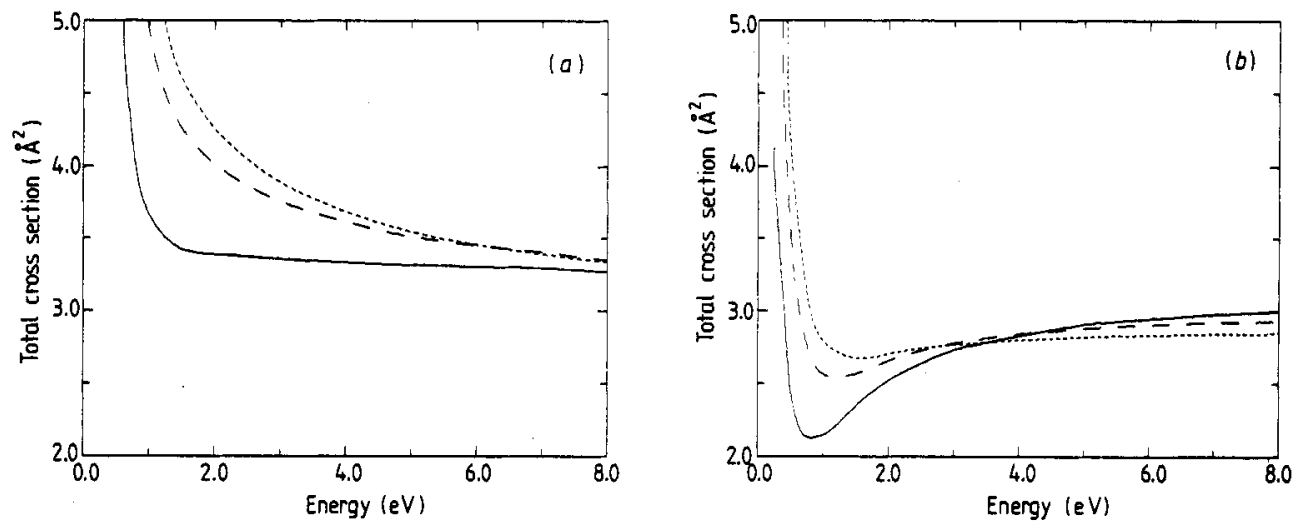

Figure 8. Total positron- $\mathrm{N}_{2}$ cross sections calculated using variously cut-off (a) ADPOS and $(b)$ ADELEC polarisation potentials: $\longrightarrow, p=1.0 ;---, p=2.0 ; \cdots, p=6.0$. For these comparisons, the cut-off radius was fixed at $r_{c}=1.59 a_{0}$ and the power parameter $p$ was varied. See also figure 9 . 

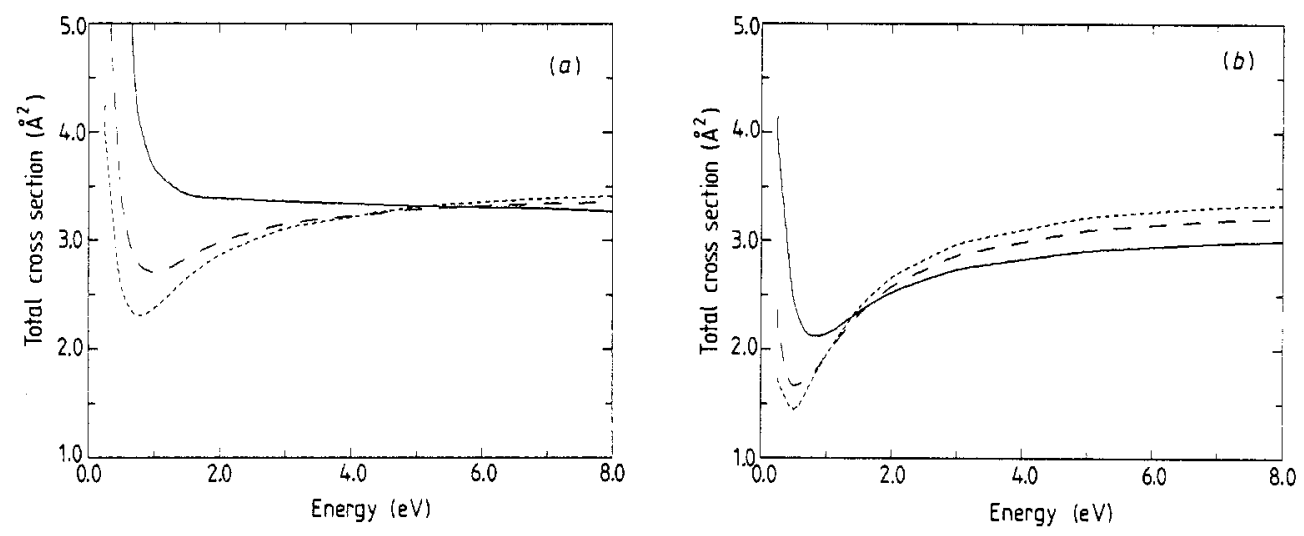

Figure 9. Total positron- $\mathrm{N}_{2}$ cross sections calculated using variously cut-off $(a)$ ADPOS and $(b)$ ADELEC polarisation potentials: $\longrightarrow, r_{\text {cut }}=1.59 ;---, r_{\text {cut }}=2.25 ; \cdots, r_{\text {cut }}=2.65$. For these comparisons, the power parameter was fixed at $p=1$ and the cut-off radius varied. See also figure 8 .

an optimum cut-off ADPOS potential, we carried out cross section studies for a wide range of values of $r_{c}$ and $p$. For a sharp cut-off (e.g., $p=6$, the value characteristic of electron-molecule systems), no value of $r_{c}$ produced cross sections that agreed even qualitatively with experimental values. Only by 'softening' the exponential in the cut-off function by using $p=1$ and thereby ensuring a gradual onset of non-adiabatic effects, could we find a cut-off radius that produced credible cross sections (see figures $8(a)$ and $9(a))$. Interestingly, use of this power parameter to cut off the ADELEC potential forced cross sections for that potential away from experiment.

This second finding suggests that in more sophisticated treatments of correlation and polarisation in positron scattering, it will be important to consider these phenomena within the context of the system at hand, rather than by reference to the corresponding electron scattering system.

The inability of a sharply cut-off polarisation potential to produce qualitatively correct positron- $\mathrm{N}_{2}$ cross sections was first noticed by Darewych (1982) in bodyframe/fixed-nuclei calculations. Rather than a fully adiabatic potential, Darewych used a cut-off asymptotic polarisation potential constructed by multiplying the asymptotic form (1) by the cut-off function (6). Using $p=6$ and varying $r_{\mathrm{c}}$, Darewych found that "no single value of [the cutoff radius] gives a shape like the observed low-energy [experimental] results over the entire energy range $E \leqslant 10 \mathrm{eV}$.' Darewych thus had to resort to an energy-dependent cut-off function obtained by adjusting $r_{\mathrm{c}}$ at each energy to ensure agreement with experiment.

In the present study, using the cut-off ADPOS potential, we were able to obtain an energy-independent cut-off function-equation (6) with $r_{\mathrm{c}}=1.59 a_{0}$ and $p=1$-that produces qualitatively correct cross sections over the entire energy range $E \leqslant 10.0 \mathrm{eV}$. (These cross sections and the corresponding eigenphase sums appear in table 2.) The essential differences between this potential and that used by Darewych are (i) the presence of adiabatic effects (which depend on the sign of the charge of the projectile) that are not a part of the (sign-independent) asymptotic form; and (ii) a more gradual onset of non-adiabatic effects as the projectile approaches the target.

The importance of proper inclusion of non-adiabatic effects is illustrated in figure 10(a), which compares cross sections for identically cut-off ADPOS and ADELEC polarisa- 
Table 2. Total and partial cross sections (in $a_{0}^{2}$ ) and eigenphase sums (in radians, shown in parentheses) for positron- $\mathrm{N}_{2}$ scattering.

\begin{tabular}{lccccc}
\hline Energy $(\mathrm{eV})$ & $\sigma\left(\Sigma_{\mathrm{g}}\right)$ & $\sigma\left(\Sigma_{\mathrm{u}}\right)$ & $\sigma\left(\Pi_{\mathrm{g}}\right)$ & $\sigma\left(\Pi_{\mathrm{u}}\right)$ & $\sigma_{\text {tot }}$ \\
\hline 0.25 & 31.689 & 4.026 & 0.140 & 0.899 & 36.771 \\
& $(0.232)$ & $(0.083)$ & $(0.013)$ & $(0.031)$ & \\
0.50 & 11.693 & 5.964 & 0.238 & 1.898 & 19.827 \\
& $(0.212)$ & $(0.143)$ & $(0.023)$ & $(0.062)$ & \\
0.75 & 4.739 & 7.017 & 0.340 & 2.651 & 14.811 \\
& $(0.178)$ & $(0.191)$ & $(0.034)$ & $(0.089)$ & \\
1.0 & 1.955 & 7.426 & 0.446 & 3.156 & 13.082 \\
& $(0.144)$ & $(0.228)$ & $(0.045)$ & $(0.112)$ & \\
1.5 & 0.703 & 7.094 & 0.663 & 3.590 & 12.232 \\
& $(0.081)$ & $(0.278)$ & $(0.066)$ & $(0.148)$ & \\
2.0 & 1.328 & 6.093 & 0.874 & 3.536 & 12.104 \\
& $(0.027)$ & $(0.304)$ & $(0.087)$ & $(0.174)$ & \\
3.0 & 3.546 & 3.924 & 1.224 & 2.851 & 11.995 \\
5.0 & $(-0.063)$ & $(0.313)$ & $(0.126)$ & $(0.201)$ & \\
& 6.868 & 1.296 & 1.522 & 1.449 & 11.847 \\
7.0 & $(-0.204)$ & $(0.267)$ & $(0.185)$ & $(0.209)$ & \\
& 8.344 & 0.414 & 1.419 & 0.756 & 11.765 \\
9.0 & $(-0.322)$ & $(0.197)$ & $(0.221)$ & $(0.192)$ & \\
& 8.774 & 0.332 & 1.151 & 0.550 & 11.658 \\
10.0 & $(-0.430)$ & $(0.125)$ & $(0.237)$ & $(0.167)$ & \\
& 8.777 & 0.428 & 1.007 & 0.547 & 11.594 \\
& $(-0.481)$ & $(0.089)$ & $(0.240)$ & $(0.153)$ & \\
\hline \multirow{4}{*}{5} & & & & & \\
& & & & &
\end{tabular}
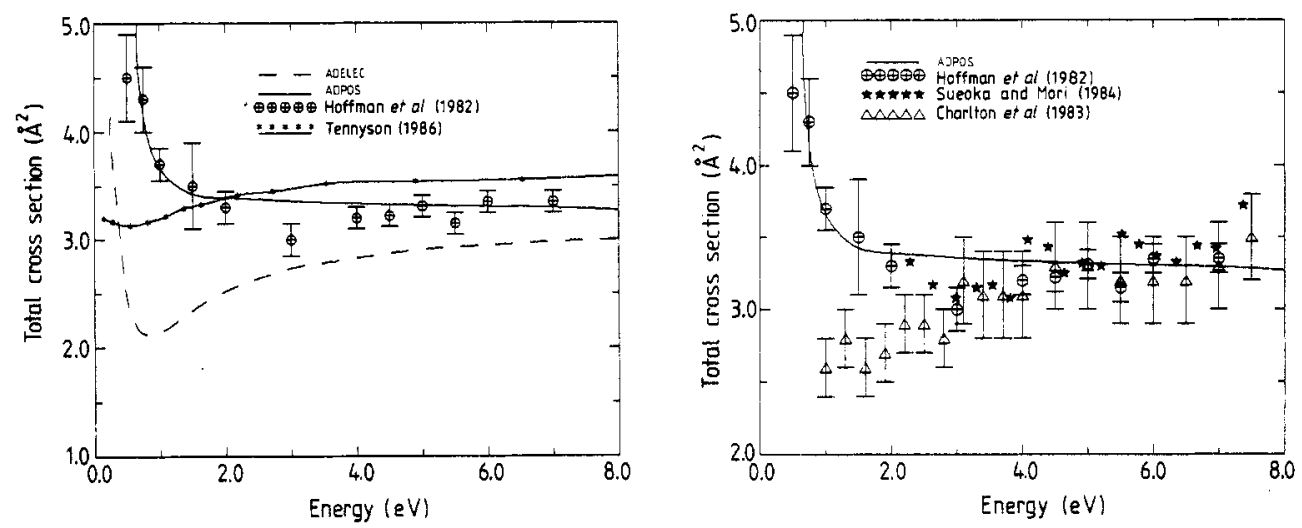

Figure 10. Total positron- $\mathrm{N}_{2}$ cross sections. (a) Comparison of identically cut-off model polarisation potentials; for each calculation, the parameters in the cut-off function of equation (6) were chosen as $p=1$ and $r_{c}=1.59 a_{0}$. Also shown are experimental data of Hoffman et al (1982) and the $R$-matrix theoretical results of Tennyson (1986). (b) Comparison of our best cut-off ADPOS results with existing experimental data.

tion potentials. For perspective, we also show in this figure the $R$-matrix results of Tennyson (1986) and the experimental data of Hoffman et al (1982). Additional experimental data (Charlton et al 1983, Suoeka and Mori 1984) are compared with our optimised cut-off ADPOS results in figure $10(b)$. In spite of the uncertainty in the various experimental results below $\sim 3 \mathrm{eV}$, these data support the veracity of the ADPOS 

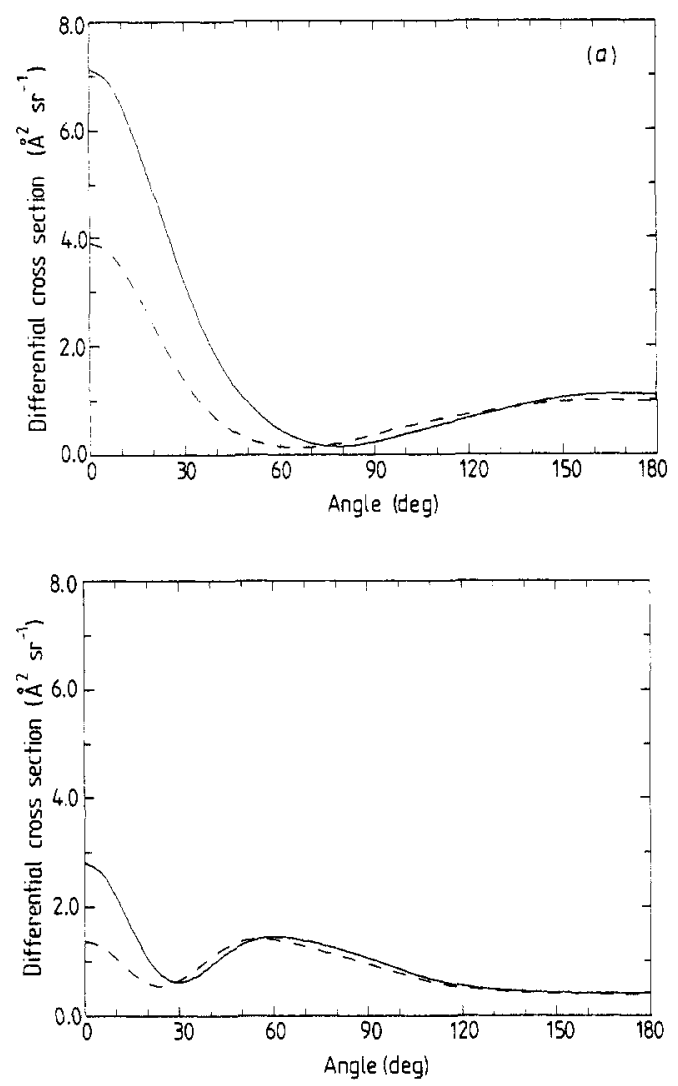

Figure 11. Total differential positron- $N_{2}$ cross sections at impact energies of $(a) 1.5 \mathrm{eV}$ and $(b) 10 \mathrm{eV}$ as calculated using identically cut-off ADELEC (- - ) and ADPOS (polarisation potentials. The parameters in the cut-off function in these calculations were $r_{\mathrm{c}}=1.59 a_{0}$ and $p=1$.

potential using a very mild cut-off function. No set of cut-off parameters $r_{\mathrm{c}}$ and $p$ could produce such agreement when either the ADELEC or asymptotic forms were used.

4.3.2. Differential cross sections. Not surprisingly, differential positron- $N_{2}$ cross sections calculated using (identically cut-off) ADPOS and ADELEC polarisation potentials are different. Interestingly, they differ primarily in the forward direction, as shown in figure 11 at a low $(1.5 \mathrm{eV})$ and a high $(10.0 \mathrm{eV})$ energy.

Apart from their intrinsic interest, the energy and angle dependences of the differential cross section provide useful guidance in the absolute normalisation of experimental positron scattering data (see, for example, Hoffman et al 1982). This information is compactly represented by the three-dimensional graph in figure 12 , which presents data calculated with the cut-off ADPOS potential that gives the total cross sections in figure 10 .

\section{Conclusions}

The graphs of total and orbital electron densities for polarised states of $\mathrm{N}_{2}(\S 4.1)$ illustrate the nature of adiabatic distortions induced by an incident positron and 


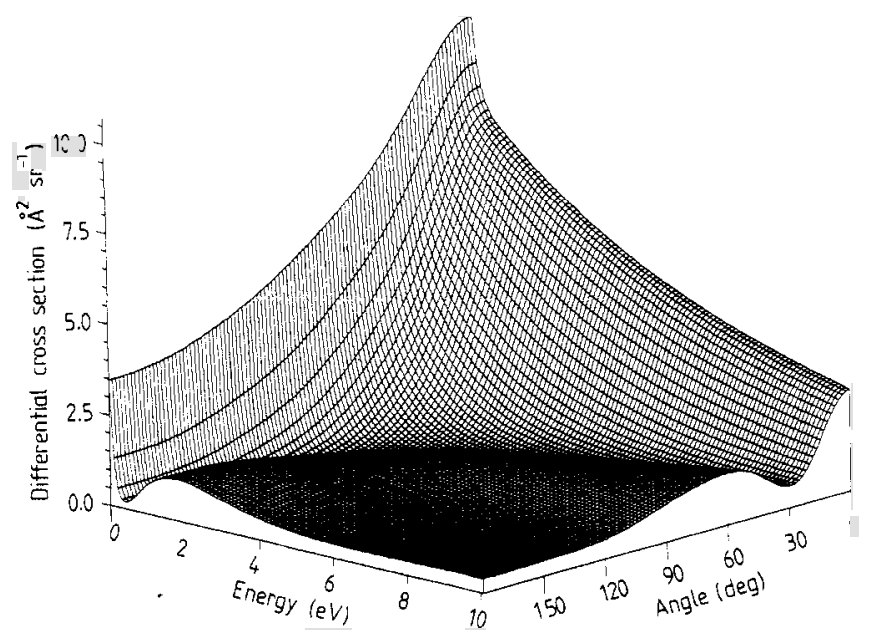

Figure 12. Total differential positron- $\mathrm{N}_{2}$ cross sections for impact energies less than or equal to $10 \mathrm{eV}$ as calculated using the cut-off ADPOS polarisation potential of figure 11 . The corresponding integrated cross sections are compared with experiment in figure $10(b)$.

demonstrate how different these distortions are from those induced by an electron. The observation that the resulting adiabatic polarisation potentials (figure 6) depend on the nature of the projectile extends to this prototypical positron-molecule system conclusions of our earlier research on scattering from $\mathrm{H}_{2}$. This aspect of positron scattering is to be expected from the high sensitivity of positron scattering cross sections to polarisation effects in general. As the potential and cross section graphs in $\S 4$ attest, non-adiabatic effects exacerbate this dependence, especially at low impact energies.

Indeed, both adiabatic and non-adiabatic effects appear to be essential to a physically realistic model of polarisation in positron scattering: only by including both could we generate cross sections in agreement with experimental data. Significantly, to do so we had to adopt a far softer cut-off than that used in electron scattering calculations. This suggests that the nature of non-adiabaticity in positron scattering is essentially different from that in electron scattering. In the latter case, velocity-dependent corrections to adiabatic polarisation effects are of crucial importance near the target; but, in positron scattering, correlation effects (which give rise to virtual positronium formation) appear gradually as the projectile nears the target, where they become dominant.

We hope that this study will contribute to the formulation of an ab initio (parameterfree) model polarisation potential for positron scattering from molecules that accurately reflects the essential physics of their interaction.

\section{Acknowledgment}

This research was supported by NSF grant PHY-8505438.

\section{References}

Alms G R, Burnham A K and Flygare W H 1975 J. Chem. Phys. 63 3321-32 Armour E A G 1984 J. Phys. B: At. Mol. Phys. 17 L375-82 
Armour E A G 1985 J. Phys. B: At. Mol. Phys. 18 3361-8

1988 Phys. Rep.

Armour E A G and Baker D J 1985 J. Phys. B: At. Mol. Phys. 18 L845-50

1986 J. Phys. B: At. Mol. Phys. 19 L871-5

1987 J. Phys. B: At. Mol. Phys. $206105-19$

Canter K F and Mills A P Jr 1982 Can. J. Phys. 60 551-64

Castillejo L, Percival I C and Seaton M J 1960 Proc. R. Soc. A 254 259-65

Charlton M, Griffith T C, Heyland G R and Wright G L 1983 J. Phys. B: At. Mol. Phys. 16 323-41

Darewych J W 1982 J. Phys. B: At. Mol. Phys. 15 L415-9

Gianturco F A, Jain A and Pantano L C 1987 Phys. Rev. A 36 4637-43

Gibson T L 1982 PhD Thesis University of Oklahoma (unpublished)

Gibson T L and Morrison M A 1984 Phys. Rev. A 29 2497-508

Herzberg G 1950 Spectra of Diatomic Molecules (New Jersey: Van Nostrand-Reinhold)

Hoffman K R, Dabaneh M S, Hsieh Y F, Kaupilla W E, Pol V, Smart J H and Stein T S 1982 Phys. Rev. A 25 1393-401

Huo W, Gibson T L, Lima M A P and McKoy V 1987 Phys. Rev. A 36 1632-44

Jain A 1986a J. Phys. B: At. Mol. Phys. 19 L105-10

1986b J. Phys. B: At. Mol. Phys. 19 L807-13

Lane N F 1980 Rev. Mod. Phys. 52 29-120

Miller T M and Bedersen B 1978 Adv. At. Mol. Phys. 13 1-55

Morrison M A 1983 Aust. J. Phys. 36 239-86

1986 Positron (Electron)-Gas Scattering ed W E Kaupilla, T S Stein and J M Wadehra (Singapore: World Scientific) pp 100-10

1988 Adv. At. Mol. Phys. 24 51-156

Morrison M A, Gibson T L and Austin D 1984 J. Phys. B: At. Mol. Phys. 17 2725-45

Morrison M A and Hay P J 1979 Phys. Rev. A $20740-8$

Morrison M A, Saha B C and Gibson T L 1987 Phys. Rev. A 36 3682-95

Nakanishi H and Schrader D M 1986a Phys. Rev. A 34 1810-22 1986b Phys. Rev. A 34 1823-40

Sueoka O and Mori S 1984 J. Phys. Soc. Japan 53 2491-2500

Schneider B I and Collins L A 1983 Phys. Rev. A 27 2847-53

Temkin A 1957 Phys. Rev. 107 1004-9

Temkin A and Vasavada K V 1967 Phys. Rev. 160 109-17

Tennyson J 1986 J. Phys. B: At. Mol. Phys. 19 4255-63

Tennyson J and Morgan L 1987 J. Phys. B: At. Mol. Phys. 20 L641-6

Truhlar D G, Dixon D A and Eades R A 1979 J. Phys. B: At. Mol. Phys. 12 1913-9 